\title{
EL TRÁNSITO A LA VIDA ADULTA DE LOS JÓVENES EN ACOGIMIENTO RESIDENCIAL: EL ROL DE LA FAMILIA
}

\author{
(YOUNG PEOPLE'S TRANSITION FROM RESIDENTIAL CARE TO \\ ADULTHOOD; THE FAMILY'S ROLE)
}

M. Elena Cuenca París Universidad Nacional de Educación a Distancia (UNED)

Gema Campos Hernando

Universidad de Alcalá (UAH)

Rosa M. Goig Martínez

Universidad Nacional de Educación a Distancia (UNED)

DOI: $10.5944 / e d u c X X 1.20201$

\section{Cómo referenciar este artículo/How to reference this article:}

Cuenca París, M. E.; Campos Hernando, G. y Goig Martínez, R. M. (2018). El tránsito a la vida adulta de los jóvenes en acogimiento residencial: El rol de la familia. Educación XX1, 21(1), 321-344, doi: 10.5944/educXX1.20201

Cuenca París, M. E.; Campos Hernando, G. \& Goig Martínez, R. M. (2018). El tránsito a la vida adulta de los jóvenes en acogimiento residencial: El rol de la familia. [Young people's transition from residential care to adulthood: The family's role]. Educación XX1, 21(1), 321-344, doi: 10.5944/educXX1.20201

\section{RESUMEN}

Este estudio es parte de una investigación más amplia sobre la puesta en marcha del plan de formación para los jóvenes protegidos en acogimiento residencial: «Plan de Preparación para la Vida Autónoma 16-21 años». Este plan, llevado a cabo por la Comunidad de Madrid, tiene como objetivo incrementar las habilidades, conocimientos y actitudes que favorezcan el proceso de autonomía y emancipación. El presente artículo se sitúa en el momento inmediatamente anterior a la salida del centro de protección, por lo que reúne información relevante sobre los riesgos y las oportunidades del tránsito a la vida adulta de los jóvenes extutelados, en concreto, aquellos relacionados con la familia de origen. Se han elaborado dos cuestionarios; uno, dirigido a los jóvenes y, otro, a los educadores de los centros. Han participado 105 
profesionales de la intervención socioeducativa y 106 jóvenes de 54 recursos de acogimiento residencial de la Dirección General de la Familia y el Menor de la Comunidad de Madrid. Los resultados plantean una contradicción entre la realidad familiar conflictiva de estos jóvenes y las expectativas de un tránsito a la vida adulta precisamente con esa familia como principal referente y recurso. Se trata de un aspecto problemático y contradictorio dado que la intervención con las familias de los jóvenes resulta compleja, la participación y el compromiso familiar con el proceso de emancipación son escasos y, en general, son valorados negativamente por los profesionales, llegando a ser considerados como un obstáculo para la transición a la vida adulta de algunos jóvenes.

\section{PALABRAS CLAVE}

Programas de transición a la vida independiente; jóvenes en dificultad social; acogimiento residencial y apoyo familiar.

\section{ABSTRACT}

This analysis is part of a broader investigation on the implementation of a training program for young people living in residential care: «Readiness for Independent Living 16-21 years.» The program, launched by the Community of Madrid, aims to increase the skills, knowledge and attitudes needed for autonomy and emancipation. This article analyzes the situation prior to the imminent departure from the residences, collecting relevant information about the risks and opportunities in the transition to adulthood of young people leaving residential care, in particular, those related to the family of origin. Two questionnaires have been developed; one designed for young people and another for educators. 105 professionals and 106 young people from 54 residential care resources belonging to the General Directorate of Family and Children of the Community of Madrid have participated in the study. The findings raise a contradiction between the conflicting family reality of these young people and the expectations for the transition to adult life, precisely with that family as the main reference and resource. This is a problematic and contradictory aspect since the intervention with the families of these young people is complex. Participation and the family's commitment to the process of emancipation are insufficient and negatively rated by the professionals, in fact, in some cases, it is considered to be an obstacle for an adequate transition to adulthood.

\section{KEY WORDS}

Transitional Programs to Independent Living; Disadvantaged Youth, Residential Care \& Family Support. 


\section{INTRODUCCIÓN}

Era difícil imaginar hace una década que adquiriría tanta relevancia el paso a la vida adulta de un colectivo de jóvenes, hasta el momento poco atendido, como eran los adolescentes acogidos en centros residenciales del sistema de protección a la infancia. A pesar de tratarse de una transición vital drástica, inusual en nuestra sociedad por la anticipación, y arriesgada en términos evolutivos, ni la legislación, ni la investigación, ni las estadísticas estatales se hacían eco del fenómeno.

Solo en el año 2013 se adoptaron en España 13.401 medidas de Acogimiento Residencial. A finales de dicho año había 1.634 menores en acogimiento residencial en la Comunidad de Madrid (Dirección General de Servicios para la Familia y la Infancia, 2014). A pesar de que el objetivo principal de estas medidas es el retorno a la familia, un 19,2\% de las bajas producidas en los centros se deben al cumplimiento de la mayoría de edad (Dirección General de Servicios para la Familia y la Infancia, 2014). Hasta el año 2013, las bajas por mayoría de edad se encontraban incluidas en la categoría «por otras causas» o por «reintegración del menor a su familia», de manera que se desconocía el número de jóvenes que afrontaba dicha transición (Dirección General de Servicios para la Familia y la Infancia, 2013), siendo las características de esta muy diferentes a las de la reinserción familiar.

En el año 2010 comenzaron a verse cambios en este ámbito. Tanto el Senado como la ONU llamaron la atención sobre este tema, indicando la necesidad de tomar medidas políticas y prácticas para facilitar el paso a la vida adulta de este colectivo. Todas estas sugerencias tomaron forma en la Ley de Modificación del Sistema de Protección a la Infancia y a la Adolescencia, aprobada el 16 de julio de 2015, que contempla en su articulado la necesidad de que las Entidades Públicas dispongan de programas y recursos destinados al apoyo y orientación de quienes, estando en acogimiento, alcanzan la mayoría de edad y quedan fuera del sistema de protección a la infancia y la adolescencia (art. 11.4).

En este marco, se está desarrollando un proyecto de investigación para el estudio del plan de formación de los jóvenes protegidos en acogimiento residencial puesto en marcha por la Comunidad de Madrid: «Plan de Preparación para la Vida Autónoma 16-21 años». El PPVA recoge las indicaciones de la investigación internacional; la preparación de dicha transición da comienzo dos años antes de la mayoría de edad (Courtney y Barth, 1996, Freundlich y Avery, 2006) y se extiende hasta los 21 años el seguimiento o acompañamiento del joven una vez emancipado (Jariot, Sala-Roca y Arnau, 2015). Con ello se pretende prevenir las situaciones marginales que sufren 
los jóvenes que se emancipan antes de los 21 años (Del Valle, Álvarez y Bravo, 2003).

Por tanto, el PPVA se dirige a los adolescentes que se encuentran acogidos en residencias de protección y que han cumplido los dieciséis años, con el objetivo de incrementar las habilidades, conocimientos y actitudes que favorezcan su proceso de autonomía y emancipación. Las diferentes áreas en las que podemos estructurar este desarrollo personal son: competencias personales, formación, integración laboral, gestión económica, vivienda y alojamiento, salud, organización doméstica, vida residencial, relaciones familiares y relaciones sociales y afectivas.

En este artículo analizamos los primeros datos obtenidos en dicha investigación. Uno de los aspectos fundamentales para facilitar una transición exitosa es conocer cómo son las relaciones sociales del joven, qué rol juega la familia de origen en el proceso de emancipación, cuál es su implicación y la relevancia que tiene para el joven en dicho proceso.

En cuanto nos introducimos en las investigaciones realizadas sobre la transición a la vida adulta, nos percatamos de que se trata de un fenómeno que se ha ido haciendo progresivamente más complejo en los últimos años en la población normalizada (Cardenal de la Nuez, 2006, Injuve, 2013, Marí-Klosé y Marí-Klosé, 2006) y que adquiere unas características muy particulares al tratarse de menores en dificultad social, la población objeto de estudio (López, Santos, Bravo y del Valle, 2013; Melendro, 2010, 2011). La principal diferencia entre ambas poblaciones a la hora de definir qué es la transición a la vida independiente reside en que cuando hablamos de jóvenes egresados del sistema de protección, esta transición tiene lugar antes de haberse realizado otras transiciones que, cultural y socialmente, la preceden; como son la finalización de los estudios básicos (Jackson y Cameron, 2012) y la consecución de un primer empleo. En el caso de los jóvenes que se emancipan desde acogimiento residencial, el cumplimiento de la mayoría de edad legal (18 años) es el requisito para que se inicie dicho proceso, estando habitualmente estas transiciones inacabadas, mientras que el resto de jóvenes adultos la realiza entre los 28 y los 48 años (Marí-Klosé y Marí-Klosé, 2006). En segundo lugar, el salto a la vida adulta se realiza, aparentemente, sin el «colchón» familiar, entendiendo como tal, el apoyo tanto económico y emocional, como de vivienda que la familia biológica provee a los jóvenes en nuestra cultura (Frimpong, 2012). Por último, el seguimiento realizado hasta el momento e, incluso, el establecido en el plan estudiado, es muy breve, dando lugar a una transición acelerada en el tiempo. Es lo que algunos autores han llamado «adultez inmediata» (Stein, 2006) o «aceleración de los procesos de autonomía», indicando que, las mencionadas características junto con las carencias experimentadas en el contexto familiar 
convierten a esta en una transición de riesgo (Campos, 2011; Freundlich, Avery y Padgett, 2007; Sala-Roca, Villalba, Jariot y Arnau, 2012; Soldevila, Peregrino, Oriol y Filella, 2013; Stein, 2005a).

\section{Vivienda o alojamiento: inestabilidad y retorno familiar}

Durante el primer año después de haberse producido la transición es cuando se hace frente a mayores dificultades (Frechon, 2003) y uno de los retos a los que se enfrentan es conseguir un hogar, un alojamiento estable en el que vivir. Se dan dos tendencias no excluyentes a la salida de los centros: numerosos cambios de alojamiento en cortos periodos de tiempo y el retorno a la familia sin ser esta una opción planificada. Aunque sabemos que existe relación entre tener un alojamiento estable y manifestar un mayor bienestar subjetivo, con independencia de las historias vividas antes y durante la estancia en protección (Cook, 1994, Wade y Dixon, 2006), sin embargo, los estudios longitudinales nos dicen que solo el 37\% de los jóvenes permanecen en el mismo alojamiento en el que se habían independizado por primera vez (Campos, 2013, Courtney, Piliavin, Grogankaylor y Nesmith, 2001), llegándose a producir hasta cinco cambios de alojamiento en periodos de uno a cuatro años (Cook, 1994). Resulta difícil definir qué es un alojamiento permanente, en cambio sí podemos decir que un alojamiento temporal lo constituyen las estancias en hoteles, hostales o casas de amigos y casi la mitad de los jóvenes $(43,5 \%)$ recurren durante ese primer año a alojamientos temporales (Biehal, Clayden, Stein y Wade, 1994).

Las cifras de retorno a la familia, aunque varían de un estudio a otro, en general son muy altas. Entre una cuarta parte y un 57\% escoge esta opción, aunque lo haga sin garantías de que sea una solución positiva o de larga duración en el tiempo (Biehal, Clayden, Stein y Wade, 1994, Campos, 2013, Freundlich y Avery, 2005, Panchón, Fernández, Vizcarro, Antón y Martín, 2001), y a pesar de que sea una opción de emancipación poco estable (Stoner, 1999).

El estudio de Tussing, Clyman y Landsverk (2001) llevado a cabo en California, compara los resultados de jóvenes que se reunificaron con sus familias después de haber estado en acogimiento familiar, con otros que no lo hicieron. Los jóvenes que regresaron a la familia mostraron más conductas auto-destructivas, más uso de sustancias, tenían con más frecuencia algún conflicto con la justicia y habían obtenido menor nivel formativo. También decían tener más problemas de conducta en general. Los autores concluyen que aquellos jóvenes que regresan a sus familias biológicas después de haber estado en acogimiento residencial tienen menor estabilidad psicológica que aquellos que no lo hacen (Tussing, Clyman y Landsverk, 2001). El éxito de 
dicha transición va a depender de la implicación de la familia en el proceso de ayuda, y del esfuerzo de los profesionales del sistema de protección (Jenson, Hawkins y Catalano, 1986).

Sin embargo, la pregunta que permanece sin respuesta es por qué lo hacen, por qué regresan a la familia cuando en todos los estudios afirman sentirse aliviados al salir de ella (Araújo y Montserrat, 2014; Jariot, SalaRoca y Arnau, 2015; Oriol, Sala-Roca, y Filella, 2014). De acuerdo don McMillen y Tucker (1999) esta opción responde a una falta de planificación adecuada en su programa educativo personal. Otra opción es que los que regresan a la familia estén peor preparados que quienes dan el paso a la vida adulta autónoma y que, por tanto, el origen de su inestabilidad no sea el contexto familiar, aunque este pudiera agravarlo (Biehal, Clayden, Stein y Wade, 1994). El retorno a la familia es negativo para la inserción socio laboral del joven cuando este sigue siendo dependiente económicamente por falta de trabajo o por falta de ahorros, cuando no ha conseguido adquirir cierta formación y cuando no ha elaborado su historia personal (Campos, 2013, Zeller y Köngeter, 2012). Por el contrario, ese retorno es positivo cuando se toma a la familia como un apoyo temporal y tienen un plan alternativo de emancipación que está apoyado o bien por la existencia de ahorros o por una inserción laboral y, finalmente, cuando consideran que han adquirido suficientes habilidades para evitar conflictos (Campos, 2013). Este último punto es relevante ya que la madurez emocional y el desarrollo físico producidos durante los años de la adolescencia puede hacer que los jóvenes sobrellevaran mejor las situaciones familiares difíciles. Pero caben otras explicaciones del fenómeno. Iglehart y Becerra (2002) sostienen que el alto porcentaje de retorno al hogar familiar que se produce sin haber solucionado los problemas previos hace pensar que los jóvenes necesitan mantener los lazos familiares para dar coherencia a su identidad con independencia de la situación familiar. En este sentido, parece que la necesidad de regresar a la familia está enraizada en una cultura en la que la pertenencia a un grupo familiar es sinónimo de normalidad (Ibrahim y Howe, 2011). Por ello, se debería hacer un esfuerzo para dotar a los jóvenes de herramientas que les permitieran sobrellevar las dificultades familiares a las que, sabemos, se van a volver a enfrentar (Iglehart y Becerra, 2002; McMillen y Tucker, 1999).

\section{La red de apoyo social y familiar durante la transición a la vida adulta}

La investigación nos indica que, en la transición a la vida adulta de los jóvenes egresados del sistema de protección, al igual que sucede con la población normalizada, la red social de apoyo es fundamental (Knorth, 
Knot-Dickscheit y Strijker, 2008; Reilly, 2003 Stein, 2005b). Estamos hablando, por tanto, de «interdependencia» más que de «independencia», entendiendo por «interdependencia» la creación y mantenimiento de redes de apoyo social con personas significativas en la vida del joven (Andrew, Williams y Waters, 2014; Flynn y Vincent, 2008; Höjer y Sjöblom, 2010; Iglehart, 1993; 1994; Kerman, Wildfire y Barth, 2002; Mendes, 2006; Reilly, 2003; Sala-Roca, Villalba, Jariot, y Arnau, 2012; Wade y Munro, 2008). Sin embargo, los resultados de la investigación en este aspecto muestran discrepancias. Por un lado, indican que los adolescentes pueden identificar dicha red sin problemas (Bravo y Fernández, 2003; Collins, 2001). Por otro lado, conocer la calidad y la trascendencia de la ayuda real que esa red de personas pueda proveer, es una labor mucho más compleja (Campos, 2013). En un estudio longitudinal realizado utilizando metodología cualitativa, los jóvenes mientras se encontraban en acogimiento, tanto familiar como residencial, decían tener altos niveles de apoyo social de amigos, de la familia acogedora o de los profesionales de protección, pero no de la familia biológica. Sin embargo, en una segunda entrevista de seguimiento que se llevó a cabo cuando ya estaban independizados, se constató que las familias biológicas jugaban un rol muy importante de apoyo emocional a la hora de ayudarles a solucionar problemas durante su transición y después de esta (Courtney, Piliavin, Grogankaylor y Nesmith, 2001). Lo anterior puede verse como un indicador de la complejidad que tienen las relaciones de los jóvenes con sus familias de origen y de las dificultades emocionales a las que algunos se enfrentan al salir de sus residencias y tener la posibilidad, o la obligación, de regresar a sus familias por no tener otra opción disponible (Montserrat, 2014; National Resource Center for Youth Development, 2004).

La red de apoyo se define como una red social que proporciona ayuda psicológica y recursos que pueden ayudar al individuo a hacer frente a sus múltiples fuentes de estrés. El apoyo social incluye aspectos como: apoyo emocional (amor y empatía) o apoyo instrumental (dinero y tiempo) (Sulimani-Aidan y Benbenishty, 2011). En este sentido, la constancia de una relación afectiva con al menos una persona de la familia, se relaciona con un mayor éxito en la transición (Stein, 2005b, Wade y Munro, 2008) y con un mayor bienestar (Freundlich, Avery y Padgett, 2007; Iglehart, 1994; Propp, Ortega y NewHeart, 2003). Mientras que otras investigaciones han constatado que los profesionales son una red ficticia con escaso peso en la transición (Stein y Verweijen-Slamnescu, 2012; Stepanova y Hackett, 2014). En un estudio realizado en Inglaterra, el 96\% de los jóvenes mantenía contacto asiduo con la familia durante su estancia en acogimiento residencial y sentía que podía solicitar su ayuda en caso de necesidad. Asimismo, las parejas y los amigos eran mencionados como fuente de ayuda. Varios trabajos ponen de ma- 
nifiesto la importancia de mantener contacto con la familia biológica; los chicos y chicas que llevaban muchos años dentro del sistema, pero habían mantenido lazos con las familias de origen, tenían menos dificultades cuando vivían de forma independiente (Courtney y Barth, 1996; Del Valle, Bravo, Martínez y Santos, 2012; Freundlich y Avery, 2006). Freundlich y Avery (2005) analizaban la relevancia de la familia de origen en la transición a la vida independiente, señalando que los adolescentes que vivían en residencias tenían menos visitas de sus familias que los que se encontraban en acogimiento familiar. Las autoras lo atribuyen a la falta de esfuerzo de los profesionales de las residencias, aunque también podría deberse a que la situación familiar de partida de los niños acogidos en familias fuera menos desestructurada que la de aquellos que reciben atención residencial. La importancia de mantener las relaciones con la familia de origen, junto con otras relaciones sociales, ha sido también descrita por los propios jóvenes al hablar de las personas relevantes en sus vidas (Mullan, McAlister, Rollock y Fitzsimons, 2007). Por último, en diferentes estudios, la mayoría de los jóvenes tenían expectativas realistas de la ayuda que sus familiares les podrían proporcionar en esta transición a la vida independiente, ya que, aunque mantenían relación con ellos, decían que no esperaban recibir su ayuda (Höjer y Sjöblom, 2010), no obstante, la relación con la familia era vista como algo necesario (Montserrat, 2014; Vinnerljung, Sundell, Andrée Löfholm y Humlesjö, 2006) y como un factor de éxito en la transición (Jones, 2012).

\section{METODOLOGÍA}

El presente estudio forma parte de un proyecto de investigación que se enmarca en el Programa de inserción sociolaboral para jóvenes en grave riesgo de exclusión social procedentes del sistema de protección a la infancia de la Comunidad de Madrid, dentro de su Plan de Autonomía 16-21.

Los objetivos de esta investigación se plantean en diferentes momentos del proyecto:

1. Inicial o de diagnóstico. En esta fase nos interesa conocer la situación y escenarios de futuro de los jóvenes de 16 años protegidos en acogimiento residencial, en el momento de su incorporación al Plan.

2. De seguimiento. En este momento, la investigación se centrará en las actuaciones y el proceso institucional.

3. De la salida. Se trata de un momento especialmente relevante en el que se consideran importantes las perspectivas y los pronósticos para la incorporación a la vida autónoma. 
4. De seguimiento continuado. Esta fase se configura desde distintos momentos a partir de los seis meses de la salida del joven del sistema de protección y hasta los cuatro años.

Para cada una de estas fases se han diseñado distintos cuestionarios que recogen información específica, en función del momento (Tabla 1).

Tabla 1

Momentos/ Instrumentos de la investigación

\begin{tabular}{ll}
\hline \multicolumn{1}{c}{ Momento } & \multicolumn{1}{c}{ Instrumento } \\
\hline Inicial o de diagnóstico & Cuestionario C1 \\
\hline Seguimiento & Cuestionario C2 \\
\hline Salida del centro de Protección & Cuestionario C3 \\
\hline Seguimiento continuado & Cuestionario C4-A los 6 meses \\
& Cuestionario C5-A l año \\
& Cuestionario C6-A los 2 años \\
& Cuestionario C7-A los 4 años \\
\hline
\end{tabular}

El estudio específico que se aborda se sitúa en el momento previo a la salida del centro de Protección (C3), cuyo objetivo fundamental era apoyar la construcción de un sistema estable de seguimiento de las trayectorias de tránsito a la vida adulta de los jóvenes extutelados de la Comunidad de Madrid. De forma operativa, se pretendía recoger información relevante, a través de las opiniones de los participantes, sobre la situación personal, laboral, formativa y económica de los jóvenes en el momento de salir del Centro con el fin de pronosticar su inclusión a la vida adulta y valorar los riesgos y las oportunidades de su emancipación de forma responsable y autónoma.

En este contexto, el artículo se circunscribe a uno de los factores más significativos en este proceso: el entorno familiar, teniendo como fin último «conocer la percepción de los jóvenes protegidos y sus educadores sobre la importancia de la familia en el momento de su emancipación».

Se elaboraron dos cuestionarios C3; uno, dirigido a los jóvenes y, otro, a los educadores de los centros. En su diseño, se contemplaron preguntas abiertas y cerradas, recogidas en ocho dimensiones: vivienda y alojamiento, relaciones familiares, sociales y afectivas, salud, formación, integración laboral y gestión económica, vida residencial y situación en el sistema de protección, competencias y expectativas y datos sociodemográficos básicos para el tratamiento estadístico de la información obtenida. 
La validación de los instrumentos se realizó a través del juicio de 10 investigadores y técnicos expertos que colaboraron en el diseño de los cuestionarios definitivos, aportando su valoración con respecto a las dimensiones de los instrumentos, su claridad y la suficiencia del contenido, para conseguir los objetivos propuestos y su adaptación a los participantes. Para ello, se elaboró un protocolo de validación, tanto para el cuestionario de jóvenes como para el de educadores.

Además, se realizó una prueba piloto, a la que respondieron 25 educadores y 28 jóvenes, para terminar de constatar si los cuestionarios eran válidos para la investigación planteada.

La aplicación de los cuestionarios se realizó en 64 recursos de acogimiento residencial de la Dirección General de la Familia y el Menor de la Comunidad de Madrid.

\section{PARTICIPANTES}

De una población $\mathrm{N}=150$ jóvenes en acogimiento residencial de la Comunidad de Madrid que iban a abandonar el sistema de protección, respondieron al cuestionario $106(70,6 \%)$. Por su parte, de una población de $\mathrm{N}=112$ educadores responsables de su intervención socioeducativa respondieron 105 (93,7\%). Por último, de los 64 centros, fueron 54 los que participaron en este estudio $(84,4 \%)$.

\section{RESULTADOS}

Someramente, se presentan los perfiles de los participantes para, después, recoger los resultados de las dimensiones relacionadas con el planteamiento teórico expuesto en la primera parte de este artículo. En concreto, el papel que juega el entorno familiar en el momento de la emancipación de los jóvenes, según sus propias valoraciones y las de sus educadores en los centros de protección.

\section{Perfil de la muestra}

Los jóvenes encuestados tienen una media de edad de 17 años. El 48,6\% son hombres y el $51,4 \%$ son mujeres.

El 36,2\% son extranjeros y llevan en España entre 2 meses y 18 años, siendo la media de estancia en nuestro país de 8 años y 8 meses (rango muy 
amplio de respuestas, entre 1 y 18 años). Un 17,1\% llegaron a España solos; un $56,1 \%$ llegaron con su familia y el $26,8 \%$, aunque vinieron solos, su familia ya estaba aquí.

Por su parte, los educadores tienen entre 24 y 59 años. El 78,1\% son mujeres y el $21,9 \%$ son hombres.

El 34,8\% están titulados en Educación Social; el 21,3\% en Psicología; el 18\% en Pedagogía; el 13,5\% en Trabajo social; el 10,1\% en Magisterio y el 2,2\% en Psicopedagogía. El resto de los profesionales cuentan con titulaciones en Sociología, Derecho y Filosofía. El 91,3\% ejercen como educadores, mientras que el 8,7\% desempeñan su labor desde Equipos Directivos, con una media de 11 años de experiencia en este ámbito.

Con respecto a la intervención socioeducativa con el joven, llevan una media de casi 3 años de intervención con cada uno de ellos.

Los resultados más relevantes sobre el rol familiar en el momento de la emancipación de los jóvenes extutelados de la Comunidad de Madrid se encuentran, fundamentalmente, en tres dimensiones del cuestionario, a saber, Relaciones familiares, sociales y afectivas; Viviendal Alojamiento y Competencias y Expectativas.

A continuación, se presentan los datos y la información obtenida en cada una de ellas.

\section{Dimensión «Relaciones familiares, sociales y afectivas»}

Los datos analizados sobre el sistema familiar de los jóvenes encuestados indican que el padre está ausente en el 29,5\% de los casos, frente al 11,4\% de los casos en los que es la madre la que está ausente; en el 26,7\% los padres están separados o divorciados con responsabilidad compartida; en el 13,3\% los padres viven juntos; en el 7,6\% de los casos los jóvenes son huérfanos de madre; en el $6,7 \%$ son huérfanos de padre y en el $4,8 \%$ son huérfanos de ambos.

En relación al apoyo que el joven puede tener de cara a su salida del centro, la tabla 2 muestra los resultados de los dos grupos encuestados. Se puede apreciar que los mayores porcentajes se encuentran en las posibilidades de apoyo a la salida del joven por parte del entorno familiar. 
Tabla 2

Posibilidades de apoyo al joven a la salida del centro. (C3J y C3E)

\begin{tabular}{lcc}
\hline \multicolumn{1}{c}{ Apoyo a la salida del centro } & Educadores (\%) & Jóvenes (\%) \\
\hline Padre y/o madre & 36,3 & 31,7 \\
\hline Otros familiares (tíos, abuelos, primos) & 13,7 & 14,4 \\
\hline Hermanos & 11,8 & 14,4 \\
\hline $\begin{array}{l}\text { Algún educador o profesional que trabaja en el } \\
\text { centro }\end{array}$ & 10,8 & 4,8 \\
\hline No cuenta con ningún apoyo & 9,8 & 6,8 \\
\hline $\begin{array}{l}\text { Otros adultos (familiares de amigos, familiares } \\
\text { de la pareja, educadores) }\end{array}$ & 6,8 & 3,8 \\
\hline Amigos del joven & 2,9 & 10,6 \\
\hline Algún educador o profesional de fuera del centro & 2,0 & 1,9 \\
\hline La propia pareja & 2,0 & 4,8 \\
\hline Pareja de la madre o del padre & 1,0 & 0 \\
\hline No sabe si cuenta con algún apoyo & 2,9 & 6,8 \\
\hline
\end{tabular}

Una vez manifestado el apoyo con el que cuentan a la salida, más de la mitad de los jóvenes $(53,8 \%)$ expresaron de forma abierta que su familia podía proporcionarles apoyo a nivel personal y apoyo para la emancipación.

Por su parte, los educadores opinaron que la calidad de la relación del joven con la familia era baja, siendo la media de sus valoraciones de 4,87, en una escala de 0 a 10.

Del mismo modo, el 73,5\% de los educadores manifiesta que la familia no ha participado en las actuaciones realizadas para facilitar el tránsito a la vida adulta.

\section{Dimensión «Vivienda/Alojamiento»}

Se les planteó a los dos grupos participantes, en pregunta cerrada, con quién vivirá el joven cuando salga del recurso de protección, encontrando distintos datos, si comparamos las respuestas de jóvenes y educadores. Sin embargo, los mayores porcentajes de ambos grupos se encuentran en las opciones de vivir con su familia $(28,4 \%$ a $32,7 \%)$ y en recursos de apoyo a la emancipación $(25,5 \%$ a $30,6 \%)$. No obstante, si se consideran las dos opciones relacionadas con el entorno familiar (con su familia; con otros familiares), podemos observar que los datos destacan significativamente de las demás $(39,8 \%$ y $37,2 \%$, respectivamente) (Tabla 3$)$. 
Tabla 3

Con quién van a vivir los jóvenes cuando salgan del centro (C3J y C3E)

\begin{tabular}{lcc}
\hline $\begin{array}{c}\text { Con quién vivirás cuando salgas/ } \\
\text { Con quién vivirá el joven }\end{array}$ & Educadores (\%) & Jóvenes (\%) \\
\hline Su familia (padre, madre, hermano) & 32,7 & 28,4 \\
\hline Otros familiares (tíos, abuelos, primos) & 7,1 & 8,8 \\
\hline Su pareja & 1,0 & 2,9 \\
\hline Amigos & 1,0 & 4,9 \\
\hline Compartiendo piso con personas que no conoce & 3,1 & 2,0 \\
\hline Otros adultos & 2,1 & 2,0 \\
\hline $\begin{array}{l}\text { Otros jóvenes en un recurso de apoyo a la eman- } \\
\text { cipación }\end{array}$ & 30,6 & 25,5 \\
\hline En un centro de menores como becario & 6,1 & 2,0 \\
\hline Solo en piso alquilado & 2,0 & 2,9 \\
\hline No sabe aún con quién vivirá & 14,3 & 20,6 \\
\hline
\end{tabular}

Respecto a esta decisión, el 55,7\% de los jóvenes manifiestan que la han tomado ellos solos, siendo el $84,9 \%$ los que indican que sus educadores saben con quién tienen pensado vivir cuando salgan del centro. Además, valoran con un 8 de media, en una escala de 0 a 10, que se ha tenido en cuenta su opinión sobre este tema en el centro.

Ante el alto porcentaje de jóvenes que han afirmado que sus educadores saben con quién vivirá cuando salgan del centro, resulta importante la opinión de estos sobre si el entorno familiar les parece un espacio adecuado para el joven. El 59,3\% de los educadores consideraron adecuado que el joven viviera con su familia (padre, madre, hermano), en tanto que la mitad $(50 \%)$ de estos profesionales eran de la misma opinión respecto a que vivieran con otros familiares (tíos, abuelos, primos).

Profundizando en la decisión tomada por los jóvenes, estos han valorado que su opinión se ha tenido muy en cuenta, con una media de 8,03, en una escala de 0 a 10 . Los educadores, también, valoraron el nivel de participación a este respecto y los datos arrojaron una media, igualmente alta $(7,42)$.

En esta investigación, resultaba interesante conocer en qué medida el joven era autónomo en la toma de decisiones sobre con quién vivir. Los resultados de estos análisis arrojaron ciertas diferencias significativas. Por un lado, entre los jóvenes que habían manifestado haber decidido por sí mismos vivir con la familia cuando salieran del centro (Rango promedio 
59,26 ) y los que no lo decidieron solos (Rango promedio $=46,27$ ), con una significación $=0,005$. Por otro lado, y contrastando con lo anterior, entre los jóvenes que habían decidido por sí mismos vivir en otro centro $(49,28)$ y los que habían tomado esta misma decisión con apoyo de los educadores $(58,80)$, con una significación algo menor $(0,042)$. Por lo tanto, se aprecia que los educadores han estado más presentes ante la decisión de vivir en otros centros de apoyo a la emancipación.

\section{Dimensión «Competencias y expectativas»}

Cuando se plantea la pregunta ¿qué piensas que será lo mejor, lo más positivo para ti cuando salgas del centro?, los jóvenes no dudan en contestar que lo mejor es ser autónomo, independiente $(23 \%)$ y sentirse libre para tomar sus propias decisiones $(18,8 \%)$; pero, también, destacan estar con la familia $(13,2 \%)$, incluso por encima de los que indican encontrar un trabajo $(10,3 \%)$ o estudiar $(9,4 \%)$.

A los educadores se les solicitó que señalaran tres indicadores que les hicieran pensar que el joven pudiera realizar un tránsito a la vida adulta independiente y responsable. Los resultados pusieron de manifiesto que el apoyo familiar era un buen indicador, solo por debajo de los valores y habilidades adquiridas en el centro. Sin embargo, cuando se trata de identificar los obstáculos que impedirían dicha transición, las relaciones familiares no estarían entre los tres grandes obstáculos, sino que aparecen en sexto lugar por debajo de la falta de habilidades, enfermedad, falta de control en los impulsos, la falta de recursos económicos y la falta de empleo.

En cuanto a la expectativa de futuro al salir del centro, el 63,2\% de los jóvenes indica que estarán mejor cuando salgan por la adquisición de independencia y poder estar de nuevo con su entorno familiar.

Por último, se presenta en la tabla 4 un análisis factorial de las puntuaciones de los educadores en las distintas áreas de intervención educativa llevadas a cabo en el centro. Se puede apreciar que los ítems se agrupan en cuatro factores de características comunes, El Factor $1 \mathrm{com}-$ parte la característica de ser aspectos necesarios para el tránsito a la vida adulta, como pueden ser las habilidades y las capacidades del joven; los tres ítems siguientes se han agrupado en un Factor 2 que caracteriza la vida cotidiana; y el Factor 3 reúne tres ítems, más asociados a varios aspectos sociolaborales. Además, se observa un cuarto Factor con un solo ítem. En este caso, la intervención educativa en relaciones familiares aparece de forma externa a los otros tres, al no compartir ninguna característica con los demás. 
Tabla 4

Valoración sobre las distintas áreas de intervención de los educadores. Análisis factorial. (C3E)

\begin{tabular}{lcccc}
\hline \multicolumn{1}{c}{ Áreas de intervención } & Factor 1 & Factor 2 & Factor 3 & Factor 4 \\
\hline Formación &, 832 & & & \\
Competencias personales &, 747 & & \\
Salud &, 577 & & \\
Relaciones sociales y afectivas &, 533 & & \\
Vida residencial & &, 829 & & \\
Organización doméstica & &, 797 &, 743 &, 692 \\
Vivienda / alojamiento & &, 566 &, 602 &, 804 \\
Integración laboral & & & & \\
Documentación & & & & \\
Gestión económica & & & & \\
Relaciones familiares & & & & \\
\hline
\end{tabular}

\section{DISCUSIÓN}

La familia de referencia es tomada por los propios jóvenes y los educadores como la principal alternativa para transitar a la vida adulta en consonancia con un trabajo realizado en el condado de Baltimore, Estados Unidos, que encontró que el $80 \%$ de los antiguos jóvenes protegidos estaban viviendo con sus padres o con otros familiares después de salir del sistema (Scannapieco, Schagrin y Scannapieco, 1995). Este hecho se constata aun pudiendo apreciar en los resultados obtenidos que el sistema familiar de estos jóvenes se presenta desestructurado, al reflejarse que en el 86,7\% de los casos son huérfanos de padre o madre; los progenitores están ausentes del núcleo familiar o bien están separados o divorciados.

El 53,8\% de los jóvenes protegidos creen que su familia puede servirle de ayuda cuando salgan del centro, expresando de forma abierta que pueden proporcionarles apoyo a nivel personal y apoyo para la emancipación. Esta percepción coincide con el estudio cualitativo realizado por Araújo y Montserrat (2014) en el que los jóvenes entrevistados manifiestan que la ayuda de tipo emocional que reciben al salir del Centro es proporcionada por algunos familiares. Los educadores, por su parte, valoran en grado bajo (4,8 sobre 10) la calidad de la relación de los jóvenes con sus familiares, declarando, además, que no han participado en las actuaciones realizadas desde el centro. Sin embargo, el entorno familiar lo consideran más un indicador para el tránsito a la vida adulta independiente y autónoma que un 
obstáculo. Unido a esto, es especialmente significativo que los jóvenes se sientan más partícipes de sus decisiones sobre el tránsito a la vida adulta cuando consideran que vivirán con la familia nuclear (padre, madre y/o hermanos) que cuando piensan que lo harán en un piso u otro tipo de recurso.

En este sentido, una revisión realizada en Estados Unidos concluyó que entre los factores que mejor predicen la adaptación favorable del joven a su nueva vida independiente se encuentra la percepción que este tenga del apoyo que recibe de su familia y la continuidad de este apoyo antes, durante y después de la transición a la vida independiente (Jenson, Hawkins y Catalano, 1986). Del mismo modo que estos autores admiten que las familias de los adolescentes institucionalizados se enfrentan a problemas complejos que generalmente no saben resolver y resulta muy difícil involucrarles en el proceso de transición de los hijos.

Ante las diferencias que existen entre la percepción que muestran educadores y jóvenes respecto a las expectativas ante el proceso de tránsito a la vida adulta que se va a acometer, se propone trabajar para un mayor ajuste; especialmente en lo que se refiere a la intervención en torno a la situación familiar, siendo esta un punto débil como refleja el análisis factorial realizado, al considerarse un factor externo a la intervención educativa realizada en el centro.

Aunque tener apoyo social es algo crítico en la transición a la vida adulta, la alta probabilidad de que parte de ese apoyo provenga de relaciones familiares que no siempre son positivas para el bienestar del adolescente, hace que sea fundamental entrenarle para anticiparse a los conflictos familiares y saber enfrentarse a situaciones de estrés, causadas por la propia familia, de una forma saludable, por lo que debería ser uno de los temas abordados en los programas de preparación para la autonomía (Collins, 2001; Iglehart y Becerra, 2002).

Por otra parte, destacar que este estudio plantea una contradicción fundamental, compartida tanto por jóvenes como por educadores, entre la realidad familiar conflictiva de estos jóvenes y las expectativas de un tránsito a la vida adulta precisamente con esa familia como principal referente y recurso, pues quienes regresan con sus familias se exponen a volver a tener los mismos problemas que originaron su salida de las mismas, o a recibir un trato que, tras su paso por protección, les resulta muy infantil (Dixon y Stein, 2003). Este aspecto es algo especialmente problemático; por un lado, la intervención con las familias de los jóvenes resulta muy compleja por sus propias características y, por otro, la participación y compromiso familiar en el proceso es escasa y valorada negativamente, llegando a percibirse 
como un obstáculo para el futuro de estos jóvenes (Araújo y Montserrat, 2014; Jariot, Sala-Roca y Arnau, 2015).

Por último, señalar que el presente estudio se centra en las opiniones y valoraciones de profesionales y jóvenes en acogimiento residencial, que forman parte de una red de recursos previstos dentro de un Plan específico, lo que supone una limitación, sobre todo, si se quieren generalizar resultados o replicar el estudio en otros planes o proyectos.

\section{NOTAS}

[1] El texto presentado se vincula al proyecto de investigación CTINV084/15-EVAP (Evaluación del Plan de Autonomía Personal 16-21) desarrollado por el grupo de investigación Contextos de Intervención Socioeducativa de la Facultad de Educación de la UNED (IP del proyecto: Miguel Melendro) y cofinanciado por la Dirección General de la Familia y el Menor de la Comunidad de Madrid, Fundación Isos (Instituto para la Sostenibilidad Social) y Opción 3 S.C. 


\section{REFERENCIAS BIBLIOGRÁFICAS}

Andrew, E., Williams, J. \& Waters, C. (2014). Dialectical behaviour therapy and attachment: Vehicles for the development of resilience in Young people leaving the care system. Clinical Child Psychology and Psychiatry, 19(4), 503-515.

Araújo, S. y Montserrat, C. (2014). Jóvenes que estuvieron en el sistema de protección social a la infancia: Reflexiones a partir de una investigación realizada en Girona, España. Psicología: Reflexão e Crítica, 27(1), 198-206.

Asamblea General de las Naciones Unidas. (2010). A/RES/64/142. Directrices sobre las modalidades alternativas de cuidado de los niños. Naciones Unidas. Recuperado de http://uni.cf/1llMCAr

Biehal, N., Clayden, J., Stein, M. \& Wade, J. (1994). Leaving Care in England: A Research Perspective. Children and Youth Services Review, 16(3-4), 231-254.

Bravo, A. y Fernández, J. (2003). Las redes de apoyo social de los adolescentes acogidos en residencias de protección. Un análisis comparativo con población normativa. Psicothema, 15(1), 136-142.

Campos, G. (2011). ¿Cómo se percibe a la infancia protegida?: de la normalización a la institucionalización. En D. Poveda (Ed.), Etnografías de la infancia y la adolescencia (pp. 165-190). Madrid: Los libros de la Catarata.

- (2013). Transición a la vida adulta de los jóvenes acogidos en residencias de protección [Tesis Doctoral]. Universidad Autónoma de Madrid. Departamento de Psicología Evolutiva y de la Educación.

Cardenal de la Nuez, M. E. (2006). El paso a la vida adulta: Dilemas y estra- tegias ante el empleo flexible. Madrid: Centro de Investigaciones Sociológicas.

Collins, M. E. (2001). Transition to adulthood for vulnerable youths: A review of research and implications for policy. The Social Service Review, 75(2), 271-291.

Cook, R J. (1994). Are we helping foster care youth prepare for their future? Children and Youth Services Review, 16(3-4), 213-229.

Courtney, M. E. y Barth, R. P. (1996). Pathways of older adolescents out of foster care: implications for independent living services. National Association of Social Workers, 41(1), 75-83.

Courtney, M. E., Piliavin, I., Grogankaylor, A. y Nesmith, A. (2001). Foster youths transition to adulthood: a longitudinal view of youth leaving care. Child Welfare League of America, 80(6), 685-717.

Del Valle, J. F., Álvarez, E. y Bravo, A. (2003). Evaluación de resultados a largo plazo en acogimiento residencial de protección a la infancia. Infancia y Aprendizaje, 26(2), 235-249.

Del Valle, J. F., Bravo, A., Martínez, M. y Santos, I. (2012). Estándares de calidad en acogimiento residencial especializado. EQUAR-E. Madrid: Ministerio de Sanidad, Servicios Sociales e Igualdad.

Dirección General de Servicios para la Familia y la Infancia. Ministerio de Sanidad, Servicios Sociales e Igualdad. (2014). Boletín Estadístico n. ${ }^{\circ} 16$ de Medidas de Protección a la Infancia (Datos 2013). Recuperado de http://bit. ly/2jzHNY3

- (2013). Boletín Estadístico n. ${ }^{\circ} 15$ de Medidas de Protección a la Infancia 
(Datos 2012). Recuperado de http:// bit.ly/2kofEqJ

Dixon, J. \& Stein, M. (2003). Leaving care in Scotland: The residential experience. Scottish Journal of Residential Child Care, 2(2), 7-17.

Flynn, R. J. \& Vincent, D. (2008). Canada. En M. Stein y E. R. Munro (Eds.), Young People's Transition from Care to Adulthood. London: Jessica Kingsley.

Frechon, I. (2003). Insertion sociale et familiale de jeunes femmes anciennement placées en foyer socio-éducatif [Tesis Doctoral]. Paris X - Nanterre, Francia.

Freundlich, M. \& Avery, R. J. (2005). Planning for permanency for youth in congregate care. Children and Youth Services Review, 27, 115-134.

- (2006). Transitioning from congregate care: Preparation and outcomes. Journal of child and family studies, 15(4), 507-518.

Freundlich, M., Avery, R. J. \& Padgett, D. (2007). Preparation of youth in congregate care for independent living. Child and Family Social Work, 12, 64-72.

Frimpong, K. (2012). Preparation for Young People Leaving Care: The Case of SOS Children's Village, Ghana. Child Care in Practice, 18(4), 341-356.

Höjer, I. \& Sjöblom, Y. (2010). Young people leaving care in Sweden. Child \& Family Social Work, 15, 118-127.

Ibrahim, R. W. \& Howe, D. (2011). The experience of jordanian care leavers making the transition from residential care to adulthood: The influence of a patriarchal and collectivist culture. Children and Youth Services Review, 33(12), 2469-2474.

Iglehart, A. P. (1993). Adolescents in Foster Care: Predicting Behavioral Malad- justment. Child and Adolescent Social Work Journal, 10(6), 521-531.

- (1994). Adolescents in Foster Care: Predicting Readiness for Independent Living. Children and Youth Services Review, 16(3/4), 159-169.

Iglehart, A. P. y Becerra, R. M. (2002). Hispanic and African America Youth: Life After Foster Care Emancipation. Journal of ethnic \& cultural diversity in social work, 11(1), 79-107.

Injuve (2013). Informe Juventud en España 2012. Madrid: Instituto de la Juventud. Recuperado de http://bit. ly/2kRc3P9

Jackson, S. y Cameron, C. (2012). Leaving care: Looking ahead and aiming higher. Children and Youth Services Review, 34(6), 1107-1114.

Jariot, M., Sala-Roca, J. y Arnau, L. (2015). Jóvenes tutelados y transición a la vida independiente: indicadores de éxito. Revista Española de Orientación y Psicopedagogía, 26(2) 90-103.

Jenson, J. M., Hawkins, J. D. \& Catalano, R. F. (1986). Social support in aftercare services for troubled youth. Children and Youth Services Review, 8, 323-347.

Jones, L. P. (2012). Predictors of success in residential education placement for foster youths. Children \& Schools, 34(2), 103-113.

Kerman, B., Wildfire, J. \& Barth, R. P. (2002). Outcomes for young adults who experienced foster care. Children and youth services review, 24(5), 319-344.

Knorth, E. J., Knot-Dickscheit, J. \& Strijker, J. (2008). The Netherlands. En M. Stein \& E. R. Munro (Eds.), Young People's Transitions from Care to Adulthood (pp. 132-146). London: Jessica Kingsley. 
Ley 26/2015, de 28 de julio, de Modificación del Sistema de Protección a la Infancia y a la Adolescencia. Madrid: Boletín Oficial del Estado

López, M., Santos, I., Bravo, A. y Del Valle, J. F. (2013). El proceso de transición a la vida adulta de jóvenes acogidos en el sistema de protección infantil. Anales de psicología, 29(1), 187-196.

Marí-Klose, P. y Marí-Klose, M. (2006). Edad del Cambio. Jóvenes en los circuitos de solidaridad intergeneracional. Madrid: Siglo XXI.

McMillen, J. C. \& Tucker, J. (1999). The status of older adolescents at exit from out-of-home care. Child Welfare League of America, 78(3), 339-360.

Melendro, M. (2010). El tránsito a la vida adulta de los jóvenes en dificultad social. Madrid: UNED.

- (2011). Estrategias educativas con adolescentes y jóvenes en dificultad social: El tránsito a la vida adulta en una sociedad sostenible. Madrid: UNED.

Mendes, P. (2006). From dependence to interdependence: towards better outcomes for young people leaving state care. Child Abuse Review, 15, 110-126.

Montserrat, C. (2014). The child protection system from the perspective of young people: Messages from 3 studies. Social Sciences, 3(4), 687-704.

Mullan, C., McAlister, S., Rollock, F. \& Fitzsimons, L. (2007). "Care just changes your Life»: Factors impacting upon the mental health of children and young people with experiences of care in Northern Ireland. Child Care in Practice, 13(4), 417-434.

National Resource Center for Youth Development. (2004). The Transition
Years: Serving Current and Former Foster Youth Ages Eighteen to Twenty-one. Recuperado de http://bit.ly/2knM1Gv

Oriol, X., Sala-Roca, J. y Filella, G. (2014). Emotional competences of adolescents in residential care: Analysis of emotional difficulties for intervention. Children and Youth Services Review, 44, 334-340.

Panchón, C., Fernández, J., Vizcarro, C., Antón, V. y Martín, C. (2001). Situación de menores de 16 a 18 años en centros de protección. Barcelona: Dulac.

Propp, J., Ortega, D. M. \& NewHeart, F. (2003). Independence or interdependence: Rethinking the transition from "Ward of the court» to adulthood. Families in Society, 84, 259-266.

Reilly, T. (2003). Transition from care: Status and outcomes of youth who age out of foster care. Child Welfare League of America, 82(6), 727-746.

Sala-Roca, J., Villalba, A., Jariot, M. \& Arnau, L. (2012). Socialization process and social support networks of out-ofcare youngsters. Children and Youth Services Review, 34, 1015-1023.

Scannapieco, M., Schagrin, J. \& Scannapieco, T. (1995). Independent Living Programs: Do They Make a Difference? Child and Adolescent Social Work Journal, 12(5), 381-389.

Senado (2010). Informe de la Comisión Especial de Estudio de la problemática de la adopción nacional y otros temas afines. Recuperado de http://bit. ly/2knEuHG

Soldevila, A., Peregrino, A., Oriol, X. \& Filella, G. (2013). Evaluation of residential care from the perspective of older adolescents in care. The need for a new construct: optimum professional proximity. Child \& Family Social Work, 18(3) 285-293. 
Stein, M. (2005a). Young people aging out of care: The poverty of theory. Children and Youth Services Review, 28(4), 422-434.

- (2005b). Resilience and Young People Leaving Care: Overcoming the Odds. York: Joseph Rowntree Foundation.

- (2006). Research Review: Young people leaving care. Child and Family Social Work 11, 273-279.

Stein, M. \& Verweijen-Slamnescu, R. (2012). When care ends: Lessons from peer research insights from young people on leaving care in Albania, The Czech Republic, Finland and Poland. Innsbruck: SOS Children's Villages International.

Stepanova, E. \& Hackett, S. (2014). Understanding Care Leavers in Russia: Young People's Experiences of Institutionalization. Australian Social Work, 67(1), 118-134.

Stoner, M. R. (1999). Life After Foster Care: Services and Policies for Former Foster Youth. Journal of Sociology and Social Welfare, 26(4), 159-175.

Sulimani-Aidan, Y. \& Benbenishty, R. (2011). Future expectations of adolescents in residential care in Israel. Children and Youth Services Review, 33(7), 1134-1141.
Tussing, H. N., Clyman, R. B. y Landsverk, J. (2001). Children who return home from foster care: A 6-year prospective study of behavioral health outcomes in adolescence. Pediatrics, 108(1), 108-118.

Vinnerljung, B., Sundell, K., Andrée Löfholm, C. \& Humlesjö, E. (2006). Former Stockholm child protection cases as young adults: Do outcomes differ between those that received services and those that did not? Children and Youth Services Review, 28, 59-77.

Wade, J. \& Dixon, J. (2006). Making a home, finding a job: Investigating the early housing and employment outcomes for young people leaving care. Child and Family Social Work, 11(3), 199-208.

Wade, J. \& Munro, E. R. (2008). United Kingdom. En M. Stein \& E. R. Munro (Eds.), Young People's Transitions From Care to Adulthood (pp. 209-224). London: Jessica Kingsley.

Zeller, M. \& Köngeter, S. (2012). Education in residential care and in school: A social-pedagogical perspective on the educational attainment of Young women leaving care. Children and Youth Services Review, 34(6), 1190-1196. 


\title{
PERFIL ACADÉMICO Y PROFESIONAL DE LOS AUTORES
}

M. Elena Cuenca París. Doctora en Pedagogía y Diplomada en Educación Social por la Universidad Nacional de Educación a Distancia (UNED). Profesora-Tutora del Centro Asociado Madrid Sur de la UNED. Sus líneas de investigación se centran en el ámbito de la Educación Social, vinculando los métodos de investigación y la intervención socioeducativa con personas mayores y jóvenes en dificultad social.

Gema Campos Hernando. Doctora en Psicología Evolutiva y de la Educación por la Universidad Autónoma de Madrid. Profesora de la Universidad de Alcalá de Henares. Ha investigado desde la Universidad de California, Los ángeles (UCLA). Las líneas de investigación se centran en la transición a la vida adulta desde acogimiento residencial y el impacto de las redes sociales en el desarrollo evolutivo en infancia y adolescencia.

Rosa M. Goig Martínez. Doctora en Ciencias de la Educación por la Universidad Nacional de Educación a Distancia. Profesora en el Departamento de Métodos de Investigación y Diagnóstico en Educación (MIDE I) de la Universidad Nacional de Educación a Distancia (UNED) de Madrid, donde, además, es Coordinadora de Tecnologías y Virtualización de la Facultad de Educación.

Las líneas de investigación se centran en analizar las Tecnologías de la Información y Comunicación, atención a la diversidad y jóvenes en dificultad social.

\author{
Dirección de los autores: M. Elena Cuenca París \\ C/ Marroquina, 36-8 \\ 28030 Madrid \\ E-mail: ecuencaparis@gmail.com \\ Gema Campos Hernando \\ C/ Mateo García, 28-1. ${ }^{\circ} \mathrm{D}$ \\ 28017 Madrid \\ E-mail: gema.camposhernando@gmail.com \\ Rosa M. Goig Martínez \\ UNED - Facultad de Educación \\ C/ Juan del Rosal, 14-2. ${ }^{\text {a Planta- }}$ \\ Despacho 2.16 \\ 28040 Madrid \\ E-mail: rmgoig@edu.uned.es
}


Fecha recepción del artículo: 06. Mayo. 2016

Fecha modificación artículo: 12. Septiembre. 2016

Fecha aceptación del artículo: 21. Septiembre. 2016

Fecha revisión para publicación: 31. Mayo. 2017 
